\title{
13. COMMISSION DES ECLIPSES DE SOLEIL
}

\section{Président: M. S. A. Mitcheld, Director of the Leander McCormick Observatory,}

University of Virginia, Charlottesville, Va., U.S.A.

Membres: MM. Abetti, Carroll, Curtis, F. M. da Costa Lobo, Danjon, C. R. Davidson, de la Baume Pluvinel, N. Donitch, Freundlich, Gerasimovich, S. Hirayama, Horn d'Arturo, McNally, Menzel, J. A. Miller, Minnaert, J. H. Moore, Slouka, Stetson, Stratton, Wood.

\section{RECENT ECLIPSES}

Since the date of the r935 Paris meeting two total eclipses have been successfully observed. Throughout the long path crossing Siberia and Japan the weather on June 19,1936 on the whole about lived up to predictions. On account of widely scattered clouds neighbouring expeditions had quite different luck with the weather. In contrast, the June 8, I937 eclipse was seen throughout the whole track under universally clear skies, which is all the more surprising for the reason that eclipse expeditions to the tropics usually fare badly with the weather. Stewart and Stokley in a ship at sea were able to observe the eclipse with a measured duration of 7 min. $6 \mathrm{sec}$., the longest period of totality in 1200 years.

To the naked eye the 1937 corona was circular in outline to an angular distance of one solar diameter. In addition there were many long spikes going out at various angles, the longest being two diameters in length. Looking through a hollow tube so as to protect the eye from sky illumination, a New Zealand observer on Canton Island observed one streamer extending to four solar diameters while photographs taken from the same island showed a spike extending to six diameters. From Peru at an elevation of 25,000 feet, with two-thirds of the earth's atmosphere below him, but on account of the low altitude of the eclipsed sun, with an air-mass three times that of a zenith sun at sea-level, Major Stevens, U.S.A., obtained airplane photographs showing a "globular corona" extending to one solar diameter. At greater angular distances the long pointed streamers made a gorgeous spectacle to observers on terra firma. Although all of the developed photographs showed many prominences, there were no huge masses such as occurred at the eclipses of I9I8 and I9I9, with consequent disappointment to those observers who were photographing the corona by colour processes. On Canton Island, Gardner used three different methods. The photographs showed a greener corona than was visible to the eye. Gardner, and also Michie of New Zealand, used a rotating sector to cut down the brightness of the inner corona. The results attained were none too satisfactory.

Although the time of sunspot maximum is still uncertain, it is highly probable that the I937 eclipse occurred within a year of spot maximum. In view of the fact that the maximum (and also the minimum) types of corona take place a year or more before maximum (and also minimum) of spots, the I937 corona had the shape confidently expected.

The I936 corona was nearly circular, having the value $a+b=0 \cdot 03$, in Ludendorff's notation.

\section{ECLIPSE PROBLEMS}

Many publications on eclipses have appeared in the past two years, chief among which are Mitchell's article in Handbuch der Astrophysik, Band 8, 1936 and Dyson and Woolley's Eclipses of Sun and Moon, I937. The latter is specially strong on the 
theoretical side and should be consulted by eclipse experts in planning work or in interpreting results.

Below will be summarized recent observations and outstanding problems in different phases of eclipse work.

\section{The Corona}

The Corona and Zodiacal Light. At the 1936 eclipse in Hokkaido, Honda (Nature, 140,682, r937) was seated in a light-proof bag which completely covered his head until after totality had commenced. With the help of a large black disk which blotted out a circle with radius of three solar diameters with the sun at the centre, Honda plotted the boundaries of the band of zodiacal light from close to the sun, where it had a width of $44^{\circ}$, along the ecliptic to a point about $40^{\circ}$ away, where it was cut off by clouds.

At the 1937 eclipse, at an altitude of 14,000 feet, Smiley in Peru photographed with a Schmidt camera of four inches aperture and of focal ratio $\mathrm{f} / \mathrm{I}$. In addition to the coronal streamers, the photographs showed a faint double wedge-shaped region extending along the ecliptic slightly brighter than the remaining region around the sun. In the vicinity of the sun the band of light had the smallest width, the total length on the photographs being about I2 degrees. It is important that observations of the zodiacal light be repeated photographically and also visually by trained zodiacal light observers. In these days of photography we are all of us inclined to forget that a trained eye, particularly on faint objects, can frequently obtain observations where the photographic plate may fail. If possible, quantitative measures should be made between the intensities of coronal and zodiacal light.

Polarization in the Corona. In the I935 I.A.U. report (p. 78 ) attention was called to the observations by Dufay and Grouiller obtained at the I 932 eclipse that showed that the amount of polarization is independent of wave-length and is a maximum of 26 per cent. at Io' from the sun's limb. Since it had been shown by Grotrian (Z.f. Ast. 3, I99, r93I) that the distribution of the energy in the corona is identical with that in the sun, it seemed easy to believe that electrons were the chief means whereby the light of the corona was diffused. Observations at recent eclipses are not in agreement with the findings of Dufay and Grouiller.

In advance of publication (in $A p$. $J$.) Cohn has furnished an abstract of his observations of the 1932 and 1934 eclipses. "The shape of the coronal polarization curves for short and long wave-lengths is different. The polarization in the integrated light, in the blue, and in the red increases steadily from the sun's limb toward the outer corona, whereas it reaches maxima in the violet and green and then decreases again. The polarization for short wave-lengths is higher than for long wave-lengths in the inner corona; this ratio is reversed for distances greater than $4^{\prime} \cdot 5$ from the limb; the polarization is independent of wave-length at $4^{\prime} \cdot 5$ resulting in the intersection of the polarization curves for short and long wavelengths at $4^{\prime} \cdot 5$. For a certain distance from the limb, the polarization at short wave-lengths is smaller at the poles than at the equator. The difference in polarization between the polar and equatorial corona is less at the long than at the short wave-lengths."

At the 1936 eclipse, under perfect atmospheric conditions, from a discussion of photographs in a zone between Io' and $40^{\prime}$ from the sun's limb, Zakharin (Nature, I40, 649, I937) confirmed the conclusion of Cohn that the degree of polarization is different for different regions of the spectrum and that the change of the polariza- 
tion with increasing distances from the sun's limb is different for different heliographic latitudes and depends on the structure of the corona.

At the I936 eclipse, the Polish expedition (Zonn, Acta Astr. 3, I35, I937) found that the intensity of the coronal radiation from the sun's limb outward diminishes more rapidly in red light $(6500 \mathrm{~A}$.) than in yellow (5500 A.), while the Italian expedition under Abetti found that the intensity of the blue (4500 A.) also diminishes more rapidly than the yellow. On the contrary at the same eclipse the Japanese expedition under Hagihara (Ann. Tokyo Astron. Obsy., and Ser., r, 9I and r39, I938) found that the coronal light diminishes in intensity inversely as the 3.5 power in blue light and as the 5.5 power in yellow light, the distances outward being measured from the sun's centre.

In view of much conflicting evidence, it is very important that both intensity and polarization measurements be repeated by many different observers. Heretofore there has been the uncertainty whether different forms of polarizing apparatus may not introduce effects into the final results. A great simplicity of technique has come through "polaroid", which was used both at the I936 and I937 eclipses. The polaroid screen may be introduced in the beam of light either at the objective or near the photographic plate. The latter position will probably give slightly better definition in the photographs, but the position at the objective makes an easier installation which will probably not bring harmful effects into the photographs. With colour filters and with photographic emulsions sensitive to different spectral regions, observers with relatively simple apparatus may engage in important work. Needless to state, all photographic plates should have calibration squares impressed on them.

Total light of the Corona compared with the Full Moon. The two measures of the I937 corona by the photoelectric cell are in good agreement. Richtmyer at sealevel on Canton Island found the total light of the corona 53 per cent. of the total light of the full moon, while Stebbins in Peru, at an altitude of 14,500 feet $(A p . J$. 87,225 , I938), obtained the value 47 per cent. At the eclipses of 1918 and 1925 , Kunz and Stebbins, with photoelectric cell, found the values 50 and 44 per cent., respectively. The ten most reliable values previous to 1937 give 47 per cent. as the average. The best measures give no indication of a variation in the total coronal light depending on the sunspot cycle. Some of the measures of the r 936 eclipse from illuminometer measurements appeared to show a very much brighter corona. However, when the light of the corona plus illuminated sky is measured, it is almost impossible to make adequate allowance for sky illumination.

The Corona without an Eclipse. During the past three summers, Lyot has continued his splendid observational work from Pic du Midi (C.R. 202, 392 and I259; 203, 1327, I936 and C.R. in advance of publication). He has photographed the following eleven emission lines:

$$
\begin{aligned}
& 3388 \cdot 10 \pm 0.07 \\
& 5 \mathrm{II} 6 \cdot 03 \pm 0.02 \\
& 5302.86 \pm 0.02 \\
& 5694 \cdot 42 \pm 0.07 \\
& 6374.5 \mathrm{I} \pm 0.03 \\
& 6701.83 \pm 0.03
\end{aligned}
$$

$$
\begin{array}{r}
7059.62 \pm 0.05 \\
7891.94 \pm 0.07 \\
8024.21 \pm 0.10 \\
10746.80 \pm 0.15 \\
10797.95 \pm 0.15
\end{array}
$$

The wave-lengths were obtained by a plane grating with the following dispersions: $5 \mathrm{~A}$. to $7.5 \mathrm{~A}$. per $\mathrm{mm}$. between $3300 \mathrm{~A}$. and $8500 \mathrm{~A}$., $\mathrm{r} 8 \mathrm{~A}$. per $\mathrm{mm}$. between $7500 \mathrm{~A}$. and $9800 \mathrm{~A}$. and $42 \mathrm{~A}$. per $\mathrm{mm}$. between $9800 \mathrm{~A}$. and II,000 A. 
In I937 Lyot obtained I25 spectra, I3 direct photographs of the corona and 60 metres of cinema film. The line 5694 was discovered in 1937; it appears on three spectra with a dispersion of $7.5 \mathrm{~A}$. per mm. It is fainter than $5 \mathrm{Ir} 6$, which in turn was not seen in 1935 but was photographed both in 1936 and I937. Lyot has not yet seen the line 5536, which has been observed several times in the past thirty years and which appears to have an intensity about equal to $5 \mathrm{II} 6$. The intensities of the coronal lines vary, 6702 was stronger in 1937 than in I 936 and it was more intense than 7059. The line at 8024 is generally quite feeble, but it appears on several spectra taken under good conditions.

The equivalent widths of the three lines 5303,6374 and 6702 were $0.80,0.97$ and I.07 A., respectively, the quotient of the equivalent width by the wave-length being nearly a constant. Lyot has also succeeded in the remarkable feat of photographing the 5303 line at Meudon.

Direct photographs of the corona in 1936 at 24-hour intervals show important changes in form. In I937, four good series of photographs were obtained at intervals of 4 to 6 hours. The detailed structure will permit the determination of motions in the corona, a problem that has been attempted by many observers at many different eclipses.

Spectrum of the Corona at Eclipses. At the 1936 eclipse, with a dispersion of five $60^{\circ}$ prisms, Williams (Observatory, 60, 214, I937) observed three coronal lines, 6374,6702 and $789 \mathrm{r} \cdot 3$ (Lyot's $789 \mathrm{r} \cdot 9$ ). This last line was discovered by Curtis and Burns at the I925 eclipse using a 6-inch concave grating with dispersion 8r A. per $\mathrm{mm}$. At the I937 eclipse, Mitchell used this same spectrograph and photographed this coronal line which has a greater absolute intensity than 6374 and also other lines.

At the 1936 eclipse, Sekiguti (Nature, r40, 724, 1937) and Tanaka, Koan and Kondô (Proc. Phys.-Math. Japan, 19, 693, I937) published wave-lengths of several faint lines not heretofore observed. In the region between 5000 and $7000 \mathrm{~A}$., where Lyot's spectra were photographed under the best conditions, there were eight and three coronal lines, respectively, given by the two Japanese expeditions, that were not in Lyot's spectra.

In addition to the eleven lines given by Lyot there are twelve other lines at wave-lengths shorter than $5000 \mathrm{~A}$. that have been observed at many eclipses. Consequently, the number of known emission lines in the corona is twenty-three with a number of additional faint lines that need to be verified.

At the I936 eclipse the second of the two Japanese expeditions referred to above compared the intensity of the continuous spectrum of the corona with that of the photosphere and found a similar energy distribution in the two spectra, in agreement with the findings of Grotrian (loc. cit.). However, at wave-lengths greater than $6200 \mathrm{~A}$. there is a band of absorption of much greater intensity in the corona than in the photosphere. Although the alpha band of atmospheric oxygen has its head at $6278 \mathrm{~A}$., there is evidently in the corona an additional absorption that cannot be attributed to oxygen.

At the same eclipse, Guth and Link (Mem. Czech. Astr. Assn. No. 5, 1937) found the same band in the sky spectrum during totality. The same band had been found in 1936 in the spectrum of twilight by Garrique and Link, in the spectrum of the night sky at Pic du Midi by Cabannes and Garrique (C.R. 202, I807 and 203, 484, I936) and in the spectrum of the aurora borealis by Störmer (Nature, 139, 584, 1937). In accordance with the theory of Störmer that aurorae are caused by corpuscular radiation from the sun, then it must happen that at the time of intense 
solar activity there would be a strong aurora on the side of the earth towards the sun. With the small dispersion of the 1936 eclipse spectra it is difficult to know the wave-length of the band, but it looks probable that it is the auroral band which one would expect to be strong near the time of sun-spot maximum.

Menzel reports from his 1936 spectra " an intense new continuous spectrum which appears in small localized volumes of the corona. The emission begins sharply at about 57I5 A. and continues towards the violet. Although the observed wavelength agrees closely with that of a capture spectrum of free electrons in the fifth quantum state of $\mathrm{He}^{+}$, one cannot be certain that this provisional identification is correct."

By comparing wave-lengths east and west, Lyot finds that the inner corona rotates at a speed about equal to that of the photosphere.

As yet we have not succeeded in deciphering the source of "coronium".

Problems of the Corona. There are many outstanding problems still awaiting solution. Eclipse observers welcome the excellent work of Lyot. In future eclipses, for observations of the spectrum of the corona there is still need of carefully designed spectrographs of great speed and large dispersion. For the emission lines of coronium long exposures are unnecessary. At the 1937 eclipse, Dunham had the most powerful spectrograph (virtually a duplicate of that used with the Iooinch Mount Wilson reflector) ever used at an eclipse. With smaller dispersions we need to compare the intensity of the coronal spectrum with that of the photosphere, and we need more and better spectra that will give the dark lines in the middle and outer corona that should be taken under clear skies devoid of water vapour in order to be sure that the Fraunhofer lines are coronal in origin and do not come from sunlight scattered in the earth's atmosphere. Much valuable information unquestionably will be obtained at the r940 eclipse.

\section{Chromosphere}

Recent Observations. At the eclipse of 1936, the British expedition in Hokkaido under the direction of Stratton, in spite of clouds during totality obtained excellent results that have been published in M.N. October, 1937. In Siberia, the HarvardMass. Inst. Technology expedition under the direction of Menzel secured carefully standardized and calibrated spectra with large dispersion in the spectral region 3200-10,000 A. (Harvard Annals, 105, 87, I937). Spectra were obtained both with the continuously moving plate and with the jumping-film. In Siberia, E. G. Williams found twelve new lines in the chromosphere between 9000 and ro,049 A., a region successfully photographed at eclipses for the first time.

He also photographed eight lines of the Paschen series of hydrogen. At the 1936 eclipse many other photographs were obtained with smaller dispersion.

At Canton Island, Dunham secured spectra with large dispersion and jumpingfilm. Mitchell obtained spectra with three concave gratings without slit. With the Allegheny grating, he photographed the ionized calcium triplet and also twentyfive lines of the Paschen series, the greatest wave-length attained being $8865 \mathrm{~A}$.

Recent Publications. Among the more important contributions dealing with the chromosphere the following may be mentioned: Cillie and Menzel (Harvard Circ. No. 4IO, I935) discuss the spectra obtained in I932. Information is given about: multiplet intensities and density gradients, self-reversal of hydrogen lines, a quantitative comparison of the highly excited with normal regions of the chromosphere, and the general theory of curves of growth and self-reversal of emission lines. A publication by Moore and Menzel on the spectrophotometry of the April 
28, I930 eclipse will soon appear. Mitchell has nearly completed a similar study of his October 2I, r930 spectra.

Valuable results were obtained at the I936 eclipse by Royds (M.N. 97, 692, I937) on spectra taken outside totality. St John, Evershed and others have found that in the sun the red shift for strong lines is larger and for the weak lines is smaller than the relativity shift. By photographing the spectrum of the sun's limb before and after totality more reliable observations may be made of the limb spectrum. By dividing the $\mathrm{Fe}$ lines investigated into three groups of different intensities, Royds found practically no differences in the sun minus iron arc displacements observed with or without an eclipse. Hence, the effect of scattering by the earth's atmosphere is too small to be of any significance. Even in the same multiplet the red shift varies with the intensities and at present there is no theory that explains the displacements in a satisfactory manner.

In spite of thin clouds, Thackeray $(M . N .97,672$, I937) has made an excellent contribution from photographs with superb definition at the beginning of totality as the dark Fraunhofer lines merged over into bright lines. It was found that the emission lines of neutral metals show an unexplained displacement to the violet relative to limb absorption amounting to the mean value of $0.02 \mathrm{I} \pm 0.0$ ro $\mathrm{A}$., a result which suggests that the emission lines do not share in the red shift of the limb spectrum.

Menzel and others at Harvard in $A p . J .85$ and 86 have shown that the Balmer and Paschen series of hydrogen owe their intensities to some form of excitation in addition to straight electron capture.

Thackeray finds from the I936 spectra that the intensities of the Balmer lines of hydrogen are in close agreement with the Harvard theory. Mitchell finds a similar agreement for the intensities of the Paschen series from the r937 eclipse.

At the 1936 eclipse, Guth and Link (loc. cit.) utilized the partial phases of the eclipse to measure the relative brilliance of the sun's disk from the centre to the limb. From 0.90 radius from the centre to the limb were found large systematic differences (at 0.97 radius amounting to 30 per cent.) when compared with the well-known results of Abbot.

Spectrographs for Chromosphere. A prism spectrograph has been described by Redman (M.N. 97, 668, I937) and grating spectrographs by Menzel and others (Harv. Ann. I05, 87, I937). Nearly all observers are using the jumping-film for photographing the flash spectrum with large dispersion. With a well-designed spectrograph, prism or grating, exposures of $\frac{1}{2}$ sec. are ample.

For obtaining the focus of slitless spectrographs of large dispersion, Menzel's method is to use a parabolic mirror of large aperture and focal length at least as great as that of the spectrograph. The slit is adjusted most accurately by means of auto-collimation against an optical flat, with the Foucault test applied to the slit jaws. The spectrograph is focused in the parallel light delivered from the concave mirror. Standardization can best be secured by an evaporated aluminium wedge placed along the slit and with exposure times equal to those employed at the eclipse.

\section{COMING EcLIPSES}

The next total eclipse, that of October I, I940, with a totality of 5.7 min., will be widely observed by astronomers the world over. As Brazil is as easy of access as South Africa, both from the United States and from Europe, it is hoped that the greater promise of clear skies in Africa will not entice all of the observers away from Brazil where the eclipsed sun will be at a greater altitude. As the eclipse is still 
two years in the future, reports on probable weather conditions at different observing locations will be published at a later date.

With the present unsettled conditions in the Far East, the eclipse of September 2 I, I94I, with a totality of $3.3 \mathrm{~min}$., which will be visible chiefly from China will probably be observed by few expeditions.

Expeditions for the I940 Eclipse. Several members of the Commission are planning to observe the eclipse. In the United States there are tentative plans only. The American astronomers look with envy on their British colleagues who through the Joint Permanent Eclipse Committee are enabled to make preparations long in advance of the eclipse. The present plans have been kindly communicated by Professor Stratton.

I. Carroll will go to Brazil with an interferometer and echelon spectroscope, to study the structure of coronal and chromospheric lines under high resolving power.

2. An expedition located in South Africa from the Royal Observatories at Greenwich and the Cape with the programme:

(a) In spite of the rather weak star field, to photograph for the relativity displacement. It is proposed to obtain an independent determination of scale by exposing on a suitable check field during totality and comparing both eclipse and check fields with the same fields photographed at night.

(b) To photograph the chromospheric spectrum with moving back camera and with discontinuous observations on a stationary plate and with a spectrograph as described by Redman (loc. cit.).

3. An expedition from the Solar Physics Observatory, Cambridge and the Radcliffe Observatory, Pretoria to be located in South Africa with the following programme:

(a) Chromospheric spectrum as in $2(b)$ above.

(b) Coronal spectrum in the extreme ultra-violet.

(c) Outer coronal spectrum with special attention to the Fraunhofer lines.

(d) Polarization of the light of the corona and surrounding sky.

(e) Photography of the zodiacal light.

4. Davidson and Woolley in South Africa:

(a) A comparison of the brightness of the corona and of the sun by means of multiple reflections.

(b) The polarization of the Fraunhofer spectrum of the corona.

5. Dingle, also in South Africa, will make a study of the chromospheric spectrum of the cusps during the partial phases as observed from a station well away from the central line.

6. Appleton will arrange for ionospheric observations during totality and Waterfield will photograph the corona through colour filters.

In $2(b)$, above, the plans are to investigate the change in line profiles from say $5^{\prime \prime}$ inside the limb to the high chromosphere. Exposures will be made with a jumping-film and the slit will be moved progressively at second contact from inside the limb to the high chromosphere, or, in other words, will be kept tangent to the moon's limb. At third contact the motion will be in the reversed direction.

S. A Mitchell

President of the Commission 